\title{
25 Research Square \\ G protein-coupled receptor kinase 4 as a prognostic factor for hepatocellular carcinoma
}

\section{Yunxiu Luo}

Central South University Xiangya Stomatological Hospital

\section{Shengjun Xiao}

Guilin Medical University 2nd Affiliated Hospital

Ruirui Li

Guilin Medical University Graduate School

Xiaoshan Jiang ( $\nabla$ jiangxs@glmc.edu.cn )

Second Affiliated Hospital of Guilin Medical University https://orcid.org/0000-0002-6060-0062

\section{Research article}

Keywords: G protein-coupled receptor kinase 4, hepatocellular carcinoma, prognostic, nomograms, survival time

Posted Date: October 31st, 2019

DOI: https://doi.org/10.21203/rs.2.16677/v1

License: (c) (1) This work is licensed under a Creative Commons Attribution 4.0 International License. Read Full License 


\section{Abstract}

BACKGROUND: GRK4 has been the least understood member of the GRKs and its role in cancer has been rarely investigated. We recently reported that GRK4 halts cell cycle progression and induces cellular senescence in HEK293 cells, which involoves in pathways of cellular development, proliferation, and cell death. The present study aimed at assessing the prognostic value of GRK4 in hepatocellular carcinoma (HCC).

METHODS: Expression of GRK4 was detected by immunohistochemistry in paired tumor and peritumoral tissues. In total, 126 patients with HCC from Western China were utilized as the training cohort to develop the nomogram. Eighty-nine cases from Eastern China were used to validate the model's performance.

RESULTS: GRK4 was expressed at lower levels in tumors than in peritumoral tissues in both training $(p<0.001)$ and validation cohorts $(p<0.001)$. Tumoral GRK4 intensity score, tumor type and T stage were independent prognostic factors and used to form a nomogram for predicting overall survival, which obtained a good concordance index of 0.82 and 0.77 in training and validation cohort, respectively. In the training cohort, the positive and negative prediction values with the nomogram were $83 \%$ and $75 \%$, respectively, and in the validation cohort was $100 \%$ and $52 \%$, respectively. Patients with nomogram scores greater than 32 and 78 were considered to have a high risk for OS.

CONCLUSIONS: Low GRK4 expression in tumor tissues indicates poor clinical outcomes. The nomogram including tumoral GRK4 expression would improve the predictive accuracy of OS in HCC patients.

\section{Introduction}

Hepatocellular carcinoma (HCC) is the sixth most prevalent cancer and the third leading cause of cancerrelated mortality worldwide ${ }^{[1]}$. Although hepatectomy, along with other therapies including radiation, radiofrequency ablation(RFA), and transcatheter arterial chemoembolization(TACE), have provided survival benefits for HCC patients, the frequent intrahepatic recurrence and extrahepatic progression remain challenging ${ }^{[1,2]}$. The TNM staging is a concise system widely used to predict postoperative outcomes of cancer patients but have limit utility in patients with HCC since the same TNM stage often have different clinical outcomes ${ }^{[2]}$. An applicable prognostic system for HCC remains to be determined.

G protein-coupled receptor kinases(GRKs) are a family of serine/threonine kinases. In mammals, seven GRK members have been identified so far. They are grouped into three subfamilies: the GRK1 subfamily (rhodopsin kinase subfamily, GRK1 and GRK7), the GRK2 subfamily ( $\beta$-adrenergic receptor kinase subfamily, GRK2 and GRK3) and the GRK4 subfamily(GRK4, GRK5, and GRK6) ${ }^{[3,4]}$. GRKs are involved in a wide range of cellular physiological and pathological activities by phosphorylation of the activated $G$ protein-coupled receptors(GPCRs) or through non-GPCR phosphorylation ${ }^{[3-5]}$. Certain GRK signaling pathways are closely related to the occurrence and progression of tumors ${ }^{[6,7]}$. Immunohistochemistry staining(IHS) showed that expression of GRK2 and GRK3 were significantly lower in tumor than in 
adjacent tissues, correlated with disease-free survival and overall survival(OS) in $\mathrm{HCC}$ patients ${ }^{[8,9]}$, while the GRK6 mediated a tumor growth signaling ${ }^{[10]}$.

The human GRK4 gene is composed of 16 exons and 4 splice variants(GRK4a/ $\beta / Y / \delta$ ) have been identified. GRK4 has been the least understood member of the GRKs. Unlike the widespread expressions of GRK2, GRK3, GRK5 and GRK6, GRK4 is limited to the testes, myometrium, kidney and brain ${ }^{[11]}$. The biological function of GRK4 involves the desensitization of LH, FSH, mGlu, GABA(B), dopamine D1 and angiotensin type 1 receptors ${ }^{[11-13]}$. GRK4 has been linked to the etiopathogenesis of essential hypertension ${ }^{[13,15]}$. Two studies showed heterogenous expression for the differential GRK4 splice variant isoforms in human invasive breast cancer and granulosa cell tumors ${ }^{[16,17]}$. We recently reported that exogenous expression of GRK4a halts cell cycle progression and induces cellular senescence in HEK293 cells ${ }^{[18]}$, which involves in pathways of cellular development, proliferation, apoptosis, aging and cell $\operatorname{death}^{[19]}$.

In this study, we investigated the distribution of full-length GRK4 (GRK4a, referred as GRK4 in this manuscript) in intratumor and peritumor tissues and their significance to prognosis for patients with HCC using clinical samples. A nomogram was constructed by applying the identified factors to predict OS for HCC patients. To our knowledge, this is the first nomogram model based-on GRK4 risk stratification in cancer.

\section{Materials And Methods}

\subsection{Patients and tissue samples}

Between January 2012 and March 2017, data collected from patients who underwent hepatectomy and were histologically diagnosed with primary HCC at The Second Affiliated Hospital of Guilin Medical University ( $2^{\text {nd }}$ AHGMU; Guilin, China). The study was approved by the Institutional Ethics Committee of $2^{\text {nd }}$ AHGMU. The inclusion criteria were as follows: (1) OS was not less than three months, (2) patients received tumor resection, and (3) tumor tissues and corresponding peritumor tissue samples were available. Patients who had any preoperative anticancer treatments, extrahepatic metastases or incomplete clinical data, or undergoing liver resection after intrahepatic recurrence or metastasis were excluded. The HCC tissues and adjacent healthy tissues were formalin-fixed and paraffin-embedded for construction as a tissue microarray(TMA). The paired HCC tissue TMA from our center was used for the training cohort to develop the nomogram, and another TMA(TMA HLivH180Su10; Shanghai Outdo Biotech), which was obtained from the National Engineering Centre for Biochip at Shanghai (Shanghai, China), was used as the validation cohort.

\subsection{Clinical and pathological characteristics}


The general patient data are shown in Table 1. The clinical parameters included tumoral GRK4 expression, gender, age, histological grade, tumor anatomical location, tumor type, tumor number, the presence of portal vein tumor thrombus (PVTT), cirrhosis status, tumor diameter, and TNM stage. Additionally, the peritumoral GRK4 expression was recorded. T stage was determined according to the 7th American Joint Committee on Cancer (AJCC) staging system. The 6th AJCC staging system was used for the patients (validation cohort) whose surgery was performed before 2010.

\subsection{Immunohistochemistry (IHC) staining}

The tissue sections were dewaxed in xylene and rehydrated in gradient alcohols. The endogenous peroxidase activity was blocked with $3 \% \mathrm{H}_{2} \mathrm{O}_{2}$ for 30 minutes and the non-specific antigen epitopes were blocked with $10 \%$ normal goat serum for 30 minutes. Sections were incubated with primary antibodies (rabbit antihuman GRK4 polyclonal antibodies, Sigma-Aldrich, HPA57023, dilution, 1:100) overnight in a moist chamber at $4^{\circ} \mathrm{C}$, and then with an anti-rabbit secondary antibody (rabbit lgG H+L; Invitrogen, 1:5000) for 20 minutes. Reaction products were visualized using 3,3'-diaminobenzidine, and counterstained with haematoxylin.

\subsection{Evaluation of immunohistochemical findings}

A Leica CCD camera system (DM2500, Microsystems, Wetzlar, Germany) was used for image acquisition and processing. As in GRK4-positive cases (shown in Figure 1),, most of the epithelial cells in the HCC and paired non-cancerous hepatic tissues were stained, and the immunohistochemical score was evaluated by intensity, which was classified as negative (score $=0$ ), weak positive (score $=1$ ), positive $($ score $=2)$, or strong positive $($ score $=3)$.

\subsection{Statistical analysis}

IBM SPSS Statistics 23.0, GraphPad Prism 7.0 and EmpowerStats was used. T-tests or Mann-Whitney tests (2-tailed) were used to compare continuous variables, and the $\chi 2$ test or Fisher's exact test was used to compare categorical variables. The Kaplan-Meier method and log-rank test were used to compare survival outcomes. A multivariate Cox proportional hazards regression model was used to evaluate the independent prognostic factors for OS. The $\beta$ coefficient of the multivariate Cox regression was proportionally exchanged with a 0 - to 100-point scale for the nomogram. The highest $\beta$ coefficient was equal to 100 points. The predictive result of the nomogram was reviewed by the concordance index (C index) and calibrated with 500 bootstrap samples. Total nomogram scores of each patient were pooled and the optimal threshold value was screened using the receiver operating characteristic curve (ROC) by maximizing the Youden index. Sensitivity, specificity, predictive values, and likelihood ratios served to accurately determine the cut-off value of the nomogram. In all analyses, the nomogram was constructed 
by multivariate Cox regression with $p<0.1$; all remaining assays were considered statistically significant at $p<0.05$.

\section{Results}

\subsection{Clinicopathological features}

Data from 448 patients who underwent hepatectomy were collected. Of these, paraffin-embedded tissues from 37 patients were inadequate for inclusion in the TMA, and 86 patients had received other treatment before surgery, had hepatectomy after recurrence, or OS was shorter than three months. Tissues from 325 patients were included, which contained 126 paired tumor tissues and peritumor tissues and an additional 199 tumor tissues. Unfortunately, 199 patients whose had incomplete clinical information or met other exclusion criteria were excluded from the survival assay. For the validation cohort, the TMA was constructed using specimens from 93 cases, containing 87 paired paraffin-embedded HCC tissues and peritumoral tissues and $6 \mathrm{HCC}$ tissues without paired peritumor tissues. Four patients whose OS were less than 3 months were excluded. In summary, 126 and 89 cases met the criteria for survival assays in the training cohort and validation cohort, respectively. The baseline characteristics of the patients are provided in Table 1. The related features of the two cohorts were similar. The clinicopathologic data of 325 cases were shown in Supplementary Table 1.

\subsection{Immunohistochemical findings of GRK4 in the TMA}

To investigate the correlation between GRK4 expression and clinical/pathological features, we carried out IHS of the TMA. GRK4 was mainly localized cytoplasmic. The stromal cells were not immunostained (Figure 1).. Either in the training cohort or validation cohort, GRK4 was overexpressed in the peritumor tissue and exhibited low expression in the tumor tissue $(p<0.001)$. Moreover, the proportion of patients with GRK4-positive peritumor tissue was more than that with positive tumor tissue (Figure 2).. The average GRK4 intensity scores in tumor and peritumor tissues in the training cohort were 1.1 and 2.5, respectively $(p<0.001)$, and 1.1 and $2.3(p<0.001)$, respectively, in the validation cohort (Figure 2a).. More than $90 \%$ of patients whose peritumor tissues displayed positive GRK4 expression (Figure $2 b$ )..

\subsection{Correlations between GRK4 staining and clinicopathological features}

As shown in Supplementary Table 2 and 3, neither intratumoral nor peritumoral GRK4 expression correlated with any pathological feature in the training or validation cohort. Neither tumoral nor peritumoral GRK4 expression differed significantly between patients with high and low histopathological grade $(p=0.36)$, PVTT $(p=0.13)$, cirrhosis $(p=0.87)$, number of tumors $(p=0.95)$ or T stage $(p=0.372)$. As expected, most of the surviving patients were not those who lacked GRK4 expression $(p<0.05)$ by the last follow-up in both cohorts. Interestingly, half of the patients with high GRK4 expression in the 
peritumor tissue displayed non-staining in tumor tissues in the training cohort $(p=0.05)$. However, a different outcome was observed after information of the additional 199 cases without paired peritumor tissues were included. Therefore, the data was reclassified into two clusters on the basis of low (score 01) and high (score 2-3) tumoral GRK4 expression. Meanwhile, the other data, such as T stage, and reassayed, were merged (Table 3). GRK4 expression in tumors was correlated with histological grade $(p<0.01), \mathrm{T}$ stage $(p<0.01), \mathrm{N}$ stage $(p<0.05)$, and total stage $(p<0.01)$ (Supplementary Figure 1$).$

\subsection{Prognosis and independent prognostic factors}

The median follow-up time was 35 months (range of 10-52 months) in the training cohort and 79 months (range of 11-80 months) in the validation cohort. In the training cohort, the 1-, 3-, and 5-year survival rates were $89 \%, 42 \%$, and $16 \%$, respectively, and the corresponding rates for the validation cohort were $76 \%, 42 \%$ and $38 \%$. The median survival time was 32 months in both cohorts ( $p=0.21$ ) (Figure $3 a)$.. The median survival time and 5-year OS of patients who lacked GRK4 expression were 26 months and 3\% in the training cohort and 16 months and 22\% in the validation cohort (Supplementary Figure 2C and 2D)..

All parameters displayed in Table 1 were analyzed by univariate Cox regression; the results are reported in Table 2. T Stage, intratumoral GRK4 expression intensity, tumor type, tumor diameter, number of tumors and presence of PVTT were associated with OS. The first three factors were independent prognostic factors. Interestingly, tumoral GRK4 overexpression was identified as a protective factor. The peritumoral GRK4 was generally independent of OS, although it was highly expressed in both cohorts.

\subsection{Construction and validation of a GRK4-based predictive nomogram}

The results of the multivariate Cox regression analysis are shown in Table 3. T stage (hazard ratio[HR] $2.135,95 \%$ confidence interval $[95 \% \mathrm{Cl}] 1.520-3.000, p<0.01)$, tumor type (HR $2.10,95 \% \mathrm{Cl} 0.88-5, \mathrm{p}<0.1)$ and intratumoral GRK4 (HR 0.53, 95\% $\mathrm{Cl} 0.26-1.11, \mathrm{p}<0.05)$ were determined to be independent risk factors, and they were incorporated into a nomogram to estimate the risk for OS (Figure 4).. The nomogram performed well in the exact evaluation of the risk of 5-year OS with an AUC of 0.82, which was further assessed by index of concordance (C-index) and displayed ideal consistency for OS risk assessed with a C-index $0.82(95 \% \mathrm{Cl} 0.74-0.90)$ by internal validation using bootstrap sampling (Figure 5a).. For the external validation, the nomogram determined a c-index of $0.77(95 \% \mathrm{Cl} 0.68-0.87)$ for appraising the risk of OS, and the calibration plot presents good concordance (Figure $5 a$ and $5 b$ )..

\subsection{Risk for OS derived from nomogram scores}

The best threshold value of the overall nomogram scores was found to be 32 and 78 in the training and validation cohorts, respectively. The sensitivity, specificity, positive predictive value, and negative predictive value in the training cohort were $86 \%, 70 \%, 83 \%$ and $75 \%$, respectively, and the presence was 
$44 \%, 100 \%, 100 \%$ and $52 \%$ in the validation cohort, respectively (Table 4).. Risk stratification was carried out using the cut-off value and cases were reclassified low risk ( $\leq$ cut-off value) and high risk (>cut-off value). Risk stratification exhibited excellent discrimination compared with any of the independent prognostic factors (Figures $3 b, 3 c$ and Supplementary Figure $1 A-F$ )..

\section{Discussion}

GRKs participate in a wide range of cellular physiological and pathological activities and there are increasing evidences indicated that some of which are closely related to the occurrence and progression of tumors ${ }^{[5-7]}$. In the present study, we reported that absent expression of GRK4 in tumors was significantly associated with worse outcomes in patients with $\mathrm{HCC}(P<0.05)$. More than $30 \%$ of patients lacked GRK4 expression in tumors. Peritumoral GRK4 expression was not associated with OS. This study also indicates that a combination of tumor type (mass or diffusion) and T stage, provides more power to predict patient outcomes. To our knowledge, this is the first study that unveils the GRK4 distribution in HCC tumors as well as its prognostic significance.

The intensity scores of GRK4 staining in the cytoplasm were lower in HCC tissue than that in peritumor tissue. This differential expression in peritumor and tumor tissues might suggest a critical factor for OS in HCC patients. There is increasingly evidence supporting that activation of the GPCR signaling results from autocrine and paracrine signals as well as aberrant GPCR overexpression in tumor cells, facilitating angiogenesis and metastasis ${ }^{[20,21]}$. GRKs negatively regulates GPCR signalings and are involved in neoplasia development. For example, downregulation of GRK2, GRK3, GRK5 and GRK6 promoted tumorigenesis and metastasis in Kaposi sarcoma, basal-like breast cancer, colon cancer and medulloblastoma ${ }^{[9,22-25]}$. In granulosa cell tumors, lower GRK4a/ $\beta$ expression was observed in malignant tumors than in non-malignant tumors, whereas GRK4ץ/ठ expression was observed in all tumor samples, indicating that GRK4 isoforms may weaken follicle-stimulating hormone (FSH) receptor uncoupling and desensitization ${ }^{[7]}$. GRK4 was overexpressed in hyperfunctioning thyroid nodules (HTNs) compared with their adjacent tissues ${ }^{[27]}$. However, GRK4a failed to induce thyroid-stimulating hormone receptor (TSHR) desensitization, which may cause HTN. GRK4 was found frequently mutated in high microsatellite instability (MSI-H) colorectal tumors and HTNs and became the cause of these tumors ${ }^{[28]}$. Recently, we reported that GRK4 is capable of inducing cellular senescence in HEK293 cells ${ }^{[18]}$, and overexpression of GRK4 affects pathways of cell development, proliferation, and cell death ${ }^{[19]}$. These observations suggest a link of GRK4 to tumor biology.

The expression pattern and function of the GRK family members have been reported differed among various tumors ${ }^{[6,7,24,25]}$. In HCC, GRK3 was expressed in larger tumors and early-stage diseases, whereas GRK6 was prone to expression in smaller, moderate histological grade and metastatic tumors ${ }^{[9,10]}$. In this study, we found that tumoral GRK4 was connected to histological grade, T stage, N stage and total stage; GRK4 tended to be expressed in patients who had early-stage HCC with no lymph node involvement (Supplementary Table 1).. 
We identified that a lack of GRK4 expression was an independent risk factor for HCC patients. In HCC, more than five staging systems are applied in practice, and each system has a particularly useful window ${ }^{[29]}$. Interestingly, regardless of which staging system was applied, almost every system was linked to prognosis in a different study ${ }^{[29,30]}$. We showed that a improving nomogram that using tumoral GRK4 expression plus tumor type and T stage performed well with the AUCs of 0.82 for prognostic prediction in HCC. In this nomogram model, the performance of T stage $(\mathrm{T} 1, \mathrm{~T} 2)$ or tumoral GRK4 intensity scoring $(1,2)$ alone did not perform well(Figure 2a and Supplementary Figure 2).. However, the combined analysis of the three factors provides unexpected benefits (Figure $3 b$ and $3 c$ ).. For the clinical practice of the model, cut-off values of 32 and 78 were used in the training and validation cohorts, respectively. These values were convenient and precise since they were calculated by sensitivity, specificity, positive predictive value and negative predictive value. According to the predictive results, the nomogram might serve as an useful tool to determine whether patients will benefit from hepatectomy or clinical trials.

\section{Conclusions}

The expression of GRK4 in tumors was lower than that in peritumoral tissues. The low expression of GRK4 in tumor was related to poor OS in HCC patients. The novel nomogram model combining tumoral GRK4 expression provides a considerable assessment of OS risk.

\section{Declarations}

\section{Acknowledgments}

This research was supported by grants from the National Natural Science Foundation of China (grant \#s 31460306 and 31660327 to X. J.) and from the Natural Science Foundation of Guangxi Province, China (grant \#s 2014GXNSFAA118158 and 2017JJA130237y to X. J.). The authors would like to express their thanks to Dr Eva Jiang (Department of Obstetrics and Gynecology, Pennsylvania Hospital, Philadelphia, USA) for her helpful comments on the manuscript.

\section{Ethics approval and consent to participate}

The study was approved by the Institutional Ethics Committee of The Second Affiliated Hospital of Guilin Medical University (Guilin, China). Informed consent was obtained from all patients.

\section{Consent for publication}

All authors approved the manuscript publication. This manuscript has not been submitted in other journals. 


\section{Conflict of interest statement}

The authors declare no conflicts of interest.

\section{Declaration}

The data that support the findings of this study are available from the corresponding author, [X J], upon reasonable request.

\section{References}

1. Bray F, Ferlay J, Soerjomataram I, Siegel RL, Torre LA, Jemal A (2018) Global cancer statistics 2018: GLOBOCAN estimates of incidence and mortality worldwide for 36 cancers in 185 countries. CA Cancer J Clin 68:394-424.

2. Bruix J, Gores GJ, Mazzaferro V (2014) Hepatocellular carcinoma: clinical frontiers and perspectives. Gut 63:844-55.

3. Gurevich EV, Tesmer JJ, Mushegian A, Gurevich VV (2012) G protein-coupled receptor kinases: more than just kinases and not only for GPCRs. Pharmacol Ther 133 :40-69.

4. Gurevich VV, Gurevich EV (2019) GPCR Signaling Regulation: The Role of GRKs and Arrestins. Front Pharmacol 10:125. doi: 10.3389/fphar.2019.00125.

5. Komolov KE, Benovic JL (2018) G protein-coupled receptor kinases: Past, present and future. Cell Signal 41:17-24.

6. Yu S, Sun L, Jiao Y, Lee LTO (2018) The Role of G Protein-coupled Receptor Kinases in Cancer. Int J Biol Sci 14:189-203.

7. Sun WY, Wu JJ, Peng WT, Sun JC, Wei W (2018) The role of G protein-coupled receptor kinases in the pathology of malignant tumors. Acta Pharmacol Sin 39:1699-1705.

8. Lin S-B, Zhou L, Liang Z-Y, Zhou W-X, Jin Y (2017) Expression of GRK2 and IGF1R in hepatocellular carcinoma: clinicopathological and prognostic significance. J Clin Pathol 70:754-9

9. Jin Y, Liang ZY, Zhou WX, Zhou L. (2017) Expression and significances of G-protein-coupled receptor kinase 3 in hepatocellular carcinoma. J Cancer 8:1972-8.

10. Li Y-P (2013) GRK6 expression in patients with hepatocellular carcinoma. Asian Pac J Trop Med 6:220-3.

11. Premont RT, Macrae AD, Stoffel RH, Chung N, Pitcher JA, Ambrose C, et al (1996) Characterization of the $\mathrm{G}$ protein-coupled receptor kinase GRK4. Identification of four splice variants. J Biol Chem 271: 6403-10. 
12. Sallese M, Salvatore L, D'Urbano E, Sala G, Storto M, Launey T, et al (2000) The G-protein-coupled receptor kinase GRK4 mediates homologous desensitization of metabotropic glutamate receptor 1 . FASEB J 14:2569-80

13. .Kanaide M, Uezono Y, Matsumoto M, Hojo M, Ando Y, Sudo Y, et al (2000) Desensitization of $\mathrm{GABA}(\mathrm{B})$ receptor signaling by formation of protein complexes of GABA(B2) subunit with GRK4 or GRK5. J Cell Physiol 210:237-45.

14. Jose PA, Soares-da-Silva P, Eisner GM, Felder RA (2010) Dopamine and G protein-coupled receptor kinase 4 in the kidney: role in blood pressure regulation. Biochim Biophys Acta 1802:1259-67.

15. Felder RA, Sanada H, Xu J, Yu PY, Wang Z, Watanabe H, et al (2002) G protein-coupled receptor kinase 4 gene variants in human essential hypertension. Proc Natl Acad Sci U S A 99:3872-7.

16. Matsubayashi J, Takanashi M, Oikawa K, Fujita K, Tanaka M, Xu M, et al (2008) Expression of G protein-coupled receptor kinase 4 is associated with breast cancer tumourigenesis. J Pathol 216:317-27.

17. King DW, Steinmetz R, Wagoner HA, Hannon TS, Chen LY, Eugster EA, et al (2003) Differential expression of GRK isoforms in nonmalignant and malignant human granulosa cells. Endocrine 22:135-42.

18. Xiao P, Huang X, Huang L, Yang J, Li A, Shen K, et al (2017) G protein-coupled receptor kinase 4induced cellular senescence and its senescence-associated gene expression profiling. Exp Cell Res 360:273-80.

19. Luo Y, Huang X, Yang J, Huang L, Li R, Wu Q, Jiang X (2019) Proteomics analysis of G proteincoupled receptor kinase 4-inhibited cellular growth of HEK293 cells. J Proteomics 207:103445. doi: 10.1016/j.jprot.2019.103445.

20. Insel PA, Sriram K, Wiley SZ, Wilderman A, Katakia T, McCann T, et al (2018) GPCRomics: GPCR Expression in Cancer Cells and Tumors Identifies New, Potential Biomarkers and Therapeutic Targets. Front Pharmacol 9:431.

21. Bar-Shavit R, Maoz M, Kancharla A, Nag JK, Agranovich D, Grisaru-Granovsky S et al (2016) G Protein-Coupled Receptors in Cancer. Int J Mol Sci 17(8). pii: E1320.

22. Billard MJ, Fitzhugh DJ, Parker JS, Brozowski JM, McGinnis MW, Timoshchenko RG, et al (2016) G Protein Coupled Receptor Kinase 3 Regulates Breast Cancer Migration, Invasion, and Metastasis. PloS One 11:e0152856.

23. Wu C-C, Tsai F-M, Shyu R-Y, Tsai Y-M, Wang C-H, Jiang S-Y (2011) G protein-coupled receptor kinase 5 mediates Tazarotene-induced gene 1-induced growth suppression of human colon cancer cells. BMC Cancer 11:175.

24. Nogués L, Palacios-García J, Reglero C, Rivas V, Neves M, Ribas C, et al (2018) G protein-coupled receptor kinases (GRKs) in tumorigenesis and cancer progression: GPCR regulators and signaling hubs. Semin Cancer Biol 48:78-90.

25. Nogués L, Reglero C, Rivas V, Neves M, Penela P, Mayor F (2017) G-Protein-Coupled Receptor Kinase 2 as a Potential Modulator of the Hallmarks of Cancer. Mol Pharmacol 91:220-8. 
26. Raghuwanshi SK, Su Y, Singh V, Haynes K, Richmond A, Richardson RM (2012) The chemokine receptors CXCR1 and CXCR2 couple to distinct G protein-coupled receptor kinases to mediate and regulate leukocyte functions. J Immunol 189:2824-32.

27. Voigt C, Holzapfel H-P, Meyer S, Paschke R (2004) Increased expression of G-protein-coupled receptor kinases 3 and 4 in hyperfunctioning thyroid nodules. J Endocrinol 182:173-82.

28. El-Bchiri J, Buhard O, Penard-Lacronique V, Thomas G, Hamelin R, Duval A. (2005) Differential nonsense mediated decay of mutated mRNAs in mismatch repair deficient colorectal cancers. Hum Mol Genet 14:2435-42.

29. Hsu C-Y, Hsia C-Y, Huang Y-H, Su C-W, Lin H-C, Lee P-C, et al (2010) Selecting an optimal staging system for hepatocellular carcinoma: comparison of 5 currently used prognostic models. Cancer 116:3006-14.

30. Chen Z-H, Hong Y-F, Lin J, Li X, Wu D-H, Wen J-Y, et al (2017). Validation and ranking of seven staging systems of hepatocellular carcinoma. Oncol Lett 14:705-14.

\section{Supporting Information Listing}

- Supplementary Table 1. Correlation between the clinicopathological features and GRK4 expression in 325 HCC patients.

- Supplementary Table 2. Correlation of Tumor GRK4 and Clinicopathological Characeristics.

- Supplementary Table 3. Correlation of Peritumor GRK4 and Clinicopathological Characeristics in Training Cohort.

- Supplementary Figure 1.

- Table S2. Effect of GRK4 overexpression on senescence-related gene expression profile in HEK293 cells

\section{Tables}


Table 1. Baseline Characteristics in Both Cohorts

\begin{tabular}{|c|c|c|c|}
\hline \multirow[t]{2}{*}{ Variable } & Training cohort, $\mathrm{n}(\%)$ & Validation cohort,n(\%) & \multirow[t]{2}{*}{$P$-value } \\
\hline & $n=126$ & $\mathrm{n}=89$ & \\
\hline Follow time, median, month & 29 & 79 & 0.207 \\
\hline $\operatorname{Age} \llbracket m e a n(s d), y$ & $50.40(10.24)$ & $53.79(10.03)$ & 0.017 \\
\hline Tumor diameter, mean(sd), cm & $8.78(3.90)$ & $7.64(5.46)$ & 0.077 \\
\hline Status & & & 0.893 \\
\hline alive & $47(37.30 \%)$ & $34(38.20 \%)$ & \\
\hline dead & $79(62.70 \%)$ & $55(61.80 \%)$ & \\
\hline Gender & & & 0.454 \\
\hline Male & 109 (86.51\%) & $80(89.89 \%)$ & \\
\hline Female & $17(13.49 \%)$ & $9(10.11 \%)$ & \\
\hline Anatomic Location & & & $<0.001$ \\
\hline Left or other & $58(46.03 \%)$ & $14(15.73 \%)$ & \\
\hline Right & $68(53.97 \%)$ & $75(84.27 \%)$ & \\
\hline Number of tumors & & & 0.002 \\
\hline Politary & $91(72.22 \%)$ & $80(89.89 \%)$ & \\
\hline Multiple & $35(27.78 \%)$ & 9 (10.11\%) & \\
\hline Tumor type & & & 0.057 \\
\hline Nodular & $57(45.24 \%)$ & $52(58.43 \%)$ & \\
\hline Mass or diffusion & $69(54.76 \%)$ & $37(41.57 \%)$ & \\
\hline Cirrhosis & & & 0.081 \\
\hline No & $57(45.24 \%)$ & $51(57.30 \%)$ & \\
\hline Yes & $69(54.76 \%)$ & $38(42.70 \%)$ & \\
\hline PVTT $^{\mathrm{a}}$ & & & 0.003 \\
\hline No & $89(70.63 \%)$ & 78 (87.64\%) & \\
\hline Yes & $37(29.37 \%)$ & $11(12.36 \%)$ & \\
\hline Grade & & & 0.277 \\
\hline 1 & $19(15.08 \%)$ & $7(7.87 \%)$ & \\
\hline 2 & $92(73.02 \%)$ & $70(78.65 \%)$ & \\
\hline 3 & $15(11.90 \%)$ & $12(13.48 \%)$ & \\
\hline Stage $\mathrm{T}$ & & & 0.564 \\
\hline 1 & $42(33.33 \%)$ & 35 (39.33\%) & \\
\hline 2 & $30(23.81 \%)$ & $15(16.85 \%)$ & \\
\hline 3 & $49(38.89 \%)$ & $34(38.20 \%)$ & \\
\hline 4 & $5(3.97 \%)$ & $5(5.62 \%)$ & \\
\hline GRK4 peritumor & & & 0.087 \\
\hline negative & $3(2.38 \%)$ & $9(10.71 \%)$ & \\
\hline $1+$ & $6(4.76 \%)$ & $4(4.76 \%)$ & \\
\hline $2+$ & $43(34.13 \%)$ & $25(29.76 \%)$ & \\
\hline $3+$ & $74(58.73 \%)$ & $46(54.76 \%)$ & \\
\hline GRK4 tumor & & & 0.402 \\
\hline negative & $53(42.06 \%)$ & $40(44.94 \%)$ & \\
\hline $1+$ & 25 (19.84\%) & $20(22.47 \%)$ & \\
\hline $2+$ & $30(23.81 \%)$ & $13(14.61 \%)$ & \\
\hline $3+$ & $18(14.29 \%)$ & $16(17.98 \%)$ & \\
\hline
\end{tabular}


Table 2. Univariate Cox Regression Analysis of Factors Associated With Survival

\begin{tabular}{|c|c|c|}
\hline Variable & $\mathrm{HR}(95 \% \mathrm{CI})$ & $P$-value \\
\hline Age & $0.99(0.97,1.01) 0.2403$ & 0.24 \\
\hline Cirrhosis, no vs yes & $0.94(0.67,1.31) 0.7015$ & 0.70 \\
\hline Tumo diameter & $1.06(1.03,1.10)$ & 0.00 \\
\hline PVTT $^{\mathrm{a}}$, no vs yes & $2.39(1.63,3.49)$ & 0.00 \\
\hline Tumor type, nodular vs diffuse & $2.97(2.06,4.27)$ & 0.00 \\
\hline Number of tumors, politary vs multiple & $2.39(1.60,3.58)$ & 0.00 \\
\hline Anatomic Location,left vs right* & $0.69(0.49,0.99) 0.0444$ & 0.04 \\
\hline \multicolumn{3}{|l|}{ Stage } \\
\hline 2 vs 1 & $1.19(0.68,2.08) 0.5447$ & 0.54 \\
\hline 3 vs 1 & $4.40(2.85,6.81)$ & 0.00 \\
\hline 4 vs 1 & $10.87(5.13,23.03)$ & 0.00 \\
\hline \multicolumn{3}{|l|}{ GRK4 tumor } \\
\hline $1+$ vs negative & $0.68(0.43,1.05) 0.0837$ & 0.08 \\
\hline $2+$ vs negative & $0.60(0.37,0.98) 0.0413$ & 0.04 \\
\hline $3+$ vs negative & $0.28(0.15,0.52)$ & 0.00 \\
\hline \multicolumn{3}{|l|}{ GRK4 peritumor } \\
\hline $1+$ vs negative & $1.74(0.56,5.40) 0.3397$ & 0.34 \\
\hline $2+$ vs negative & $1.42(0.60,3.34) 0.4268$ & 0.43 \\
\hline $3+$ vs negative & $1.70(0.74,3.91) 0.2125$ & 0.21 \\
\hline \multicolumn{3}{|l|}{ Grade } \\
\hline 2 vs 1 & $1.10(0.65,1.87) 0.7218$ & 0.72 \\
\hline 3 vs 1 & $1.40(0.71,2.75) 0.3293$ & 0.33 \\
\hline
\end{tabular}

a: PVTT, portal vein tumor thrombus 
Table 3. Muilivariate Cox Regression Analysis of Factors Associated With Survival

\begin{tabular}{|c|c|c|}
\hline \multirow[t]{2}{*}{ Variable } & \multicolumn{2}{|c|}{ Multivariate } \\
\hline & HR (95\%CI) & $P$-value \\
\hline Tumo diameter & $1.04(0.96,1.13)$ & 0.31 \\
\hline PVTT $^{a}$, no vs yes & $1.07(0.52,2.20)$ & 0.85 \\
\hline Tumor type, diffuse vs nodular & $2.10(0.88,5.00)$ & 0.09 \\
\hline Number of tumors, politary vs multiple & $0.89(0.41,1.94)$ & 0.77 \\
\hline Anatomic Location, left ${ }^{\mathrm{b}}$ vs right & $0.94(0.57,1.56)$ & 0.82 \\
\hline \multicolumn{3}{|l|}{ Stage } \\
\hline 1 vs 3 & $0.77(0.32,1.85)$ & 0.04 \\
\hline 2 vs 3 & $2.81(1.02,7.72)$ & 0.01 \\
\hline 4 vs 3 & $10.75(2.57,44.97)$ & 0.02 \\
\hline \multicolumn{3}{|l|}{ GRK4 tumor } \\
\hline $1+$ vs negative & $0.50(0.26,0.97)$ & 0.04 \\
\hline $2+$ vs negative & $0.53(0.26,1.11)$ & 0.09 \\
\hline $3+$ vs negative & $0.28(0.12,0.64)$ & 0.00 \\
\hline
\end{tabular}

a: PVTT, portal vein tumor thrombus; b: left or other

Table 4. Accuracy of the Prediction Score of the Nomogram for Estimating the Risk of OS

\begin{tabular}{|c|c|c|c|c|c|c|}
\hline iable & Training & Cohort & $\left(95 \% C I^{b}\right)$ & Validation & Cohort & $(95 \% \mathrm{CI})$ \\
\hline $\mathrm{C}^{\mathrm{C}}$ & 0.82 & 0.74 & 0.90 & 0.77 & 0.68 & 0.87 \\
\hline ;-off score & & 32 & & & 78 & \\
\hline ısitivity, \% & 86 & 72 & 88 & 44 & 32 & 71 \\
\hline cificity, \% & 70 & 47 & 72 & 100 & 32 & 100 \\
\hline ;itive predictive value, $\%$ & 83 & 75 & 83 & 100 & 69 & 100 \\
\hline yative predictive value, \% & 75 & 61 & 86 & 52 & 52 & 70 \\
\hline ;itive likelihood ratio & 2.89 & 2.12 & 3.26 & 1 & 1.36 & 2.34 \\
\hline yative likelihood ratio & 0.2 & 0.23 & 0.43 & $\inf$ & 0.56 & 0.46 \\
\hline
\end{tabular}

a, OS, overall survival; b, CI, confidence interval; ROC, receiver operating characteristic curve; AUC, area under the ROC curve. ROC, receiver operating characteristic curve; 


\section{Figures}
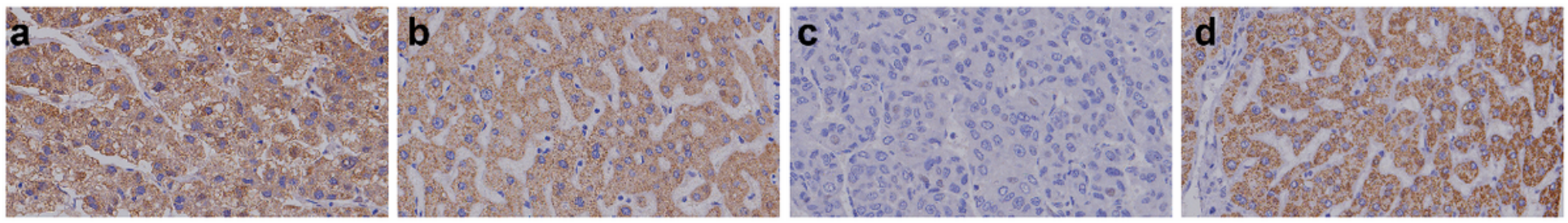

Figure 1

GRK4 expressed in paired tumor tissue and peritumor tissues in two HCC patients. It is mainly expressed in the cytoplasm and membrane both in tumor and peritumor tissues, and it was rich in peritumor tissues (enlarge 40x); a, b: GRK4 is expressed in tumor (a) and peritumor tissues (b) in case 1; c, d: GRK4 is expressed in tumor (c) and peritumor tissues (d) in case 2.
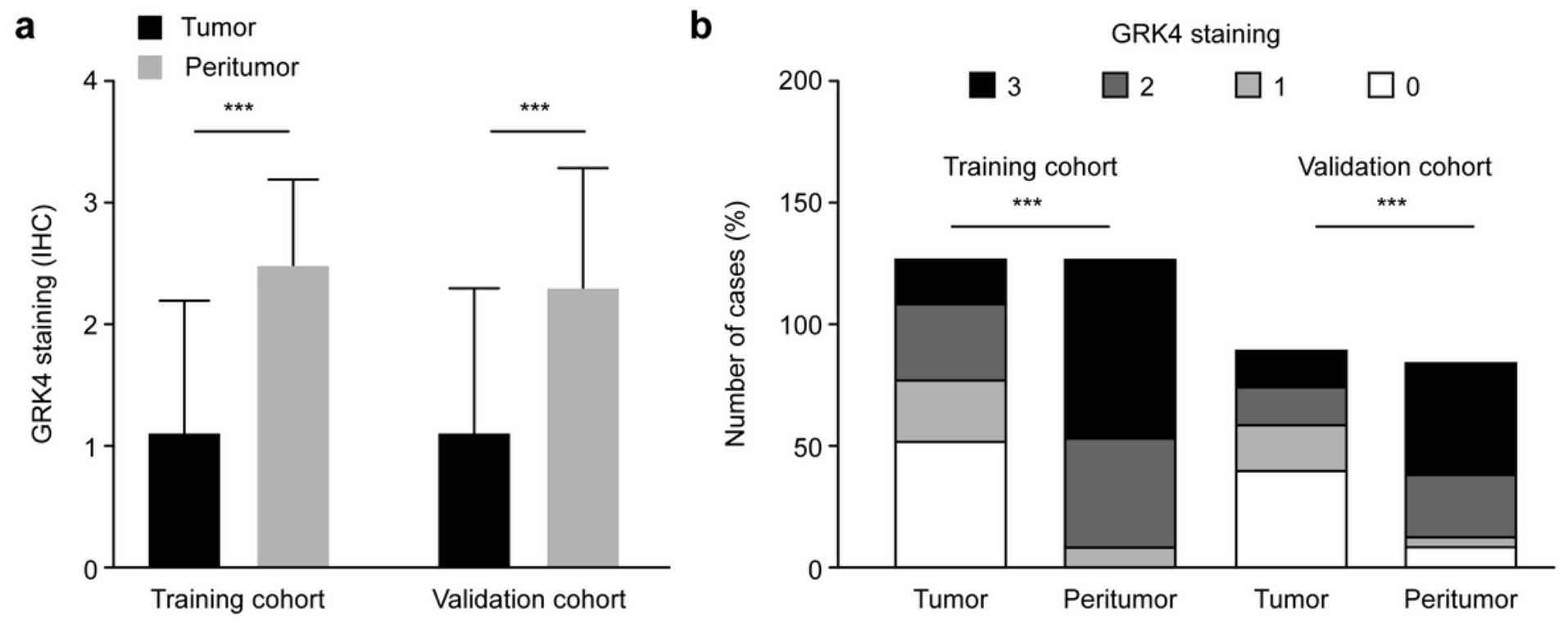

\section{Figure 2}

Expression of GRK4 staining with immunochemistry in tumor and peritumor tissues between the training and validation cohorts. a, Intensity of GRK4 staining was different between tumor and peritumor tissues. Paired sample t-test and independent sample t-test showed a statistical significance in the training cohort and validation cohort, respectively. ${ }^{\star * \star} p<0.001$. b, Distribution of various intensity scores of GRK4 staining in tumor and peritumor tissues between the cohorts. $\times 2$ test displayed a significant difference between the cohorts. $* \star \star p<0.001$. 
a

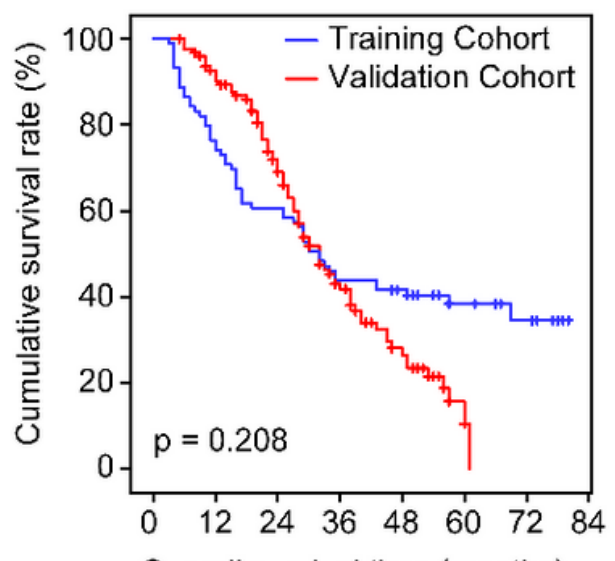

Overall survival time (months) b

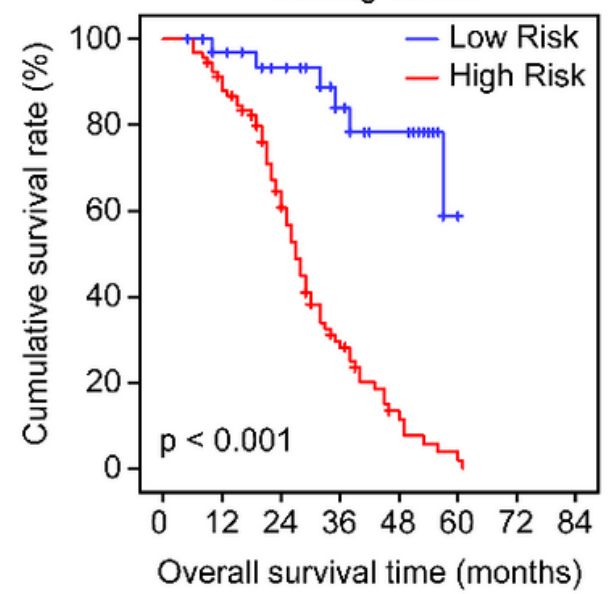

C

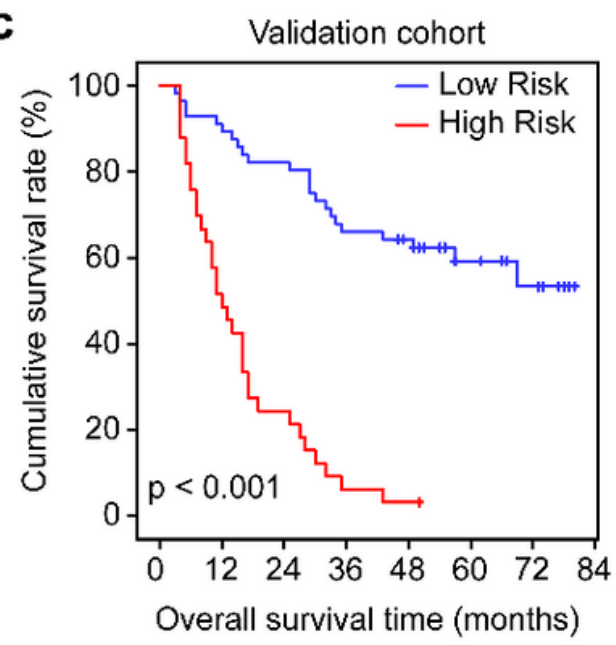

Figure 3

Cumulative overall survival (OS) curves of patients in the training cohort and validation cohort. a, OS curves of patients with HCC in the training set and validation set. The log-rank test showed that the OS was not significantly different between the two cohorts. b, OS curves of subgroups with risk stratified by dividing HCC patients into low-risk and high-risk groups based on the nomogram score cut-off value in the training cohort, which was statistically significant $(p<0.001)$. c, OS plots of the subgroups with risk stratified in the validation cohort show significant differences $(p<0.001)$. 
Points

$\begin{array}{lllllllllll}0 & 10 & 20 & 30 & 40 & 50 & 60 & 70 & 80 & 90 & 100\end{array}$

\section{Type}

GRK4Tumor

TStage

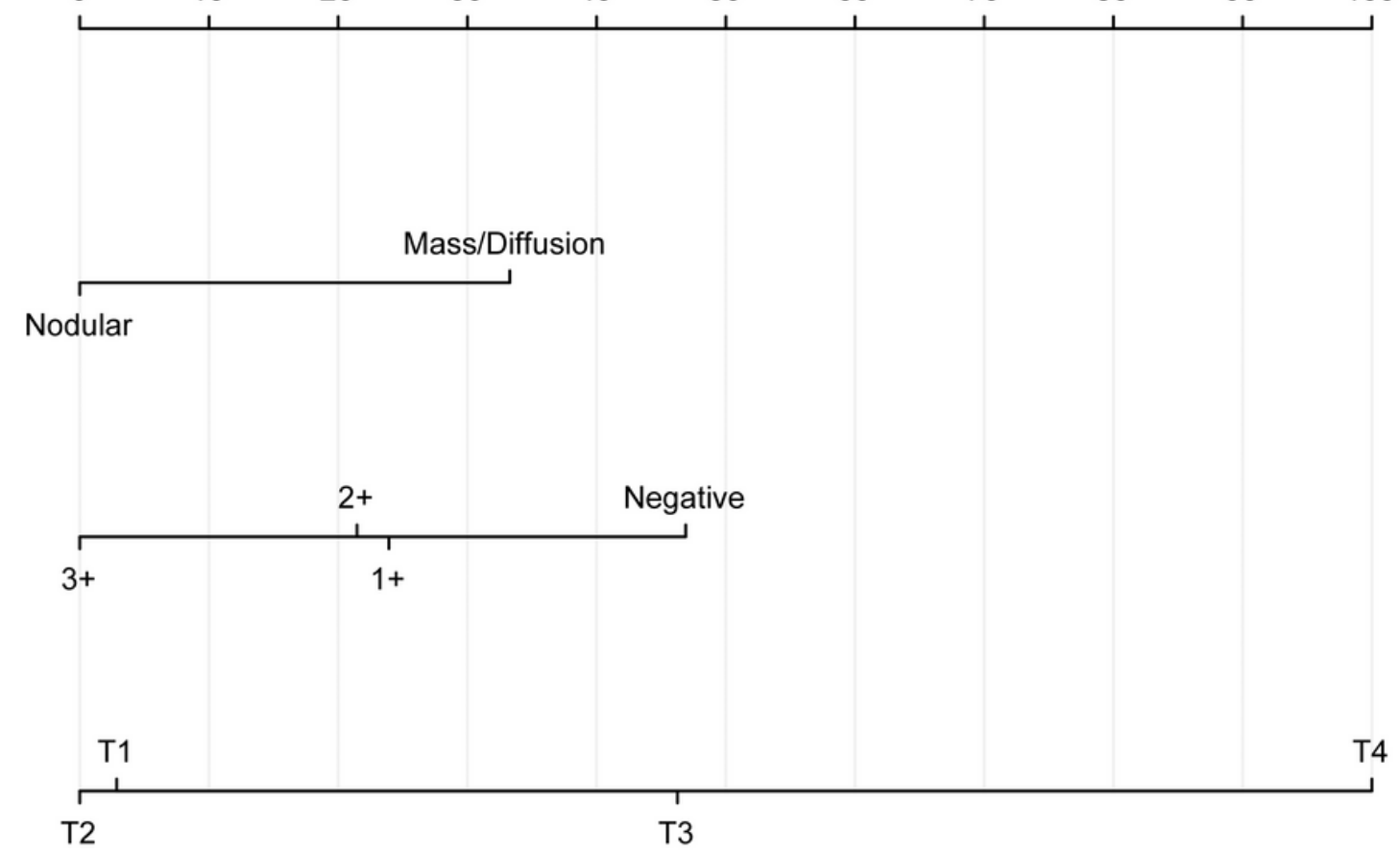

Total points

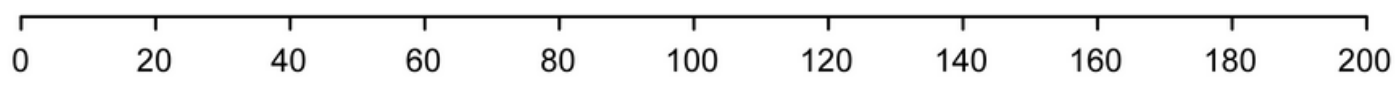

Linear predictor

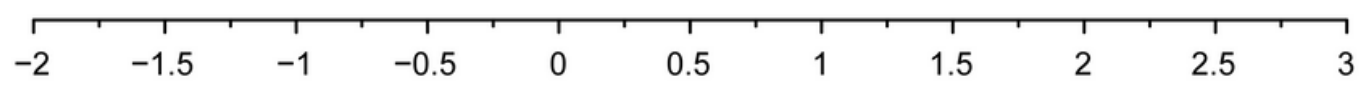

1-year survival

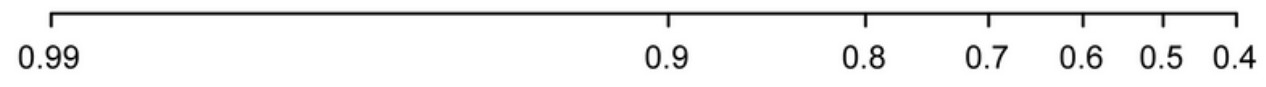

3-year survival

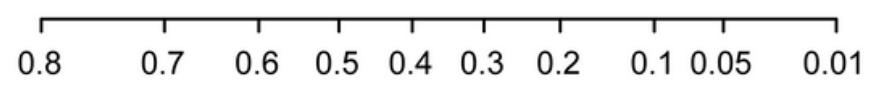

5-year survival

\begin{tabular}{lllllllll}
\hline & & & & & & & \\
0.6 & 0.5 & 0.4 & 0.3 & 0.2 & 0.1 & 0.05 & 0.01
\end{tabular}

\section{Figure 4}

Nomogram to estimate the risk for 1-, 3-, and 5-year overall survival (OS), combining GRK4 presence in tumor tissue, tumor type and $\mathrm{T}$ stage to form the nomogram. To use the nomogram, find the position of each variable on the corresponding axis, draw a line to the point value axis to determine the number of points for each variable, add the points from all of the variables, and draw a line from the total points axis to determine the probabilities at the lower line of the nomogram. 
a

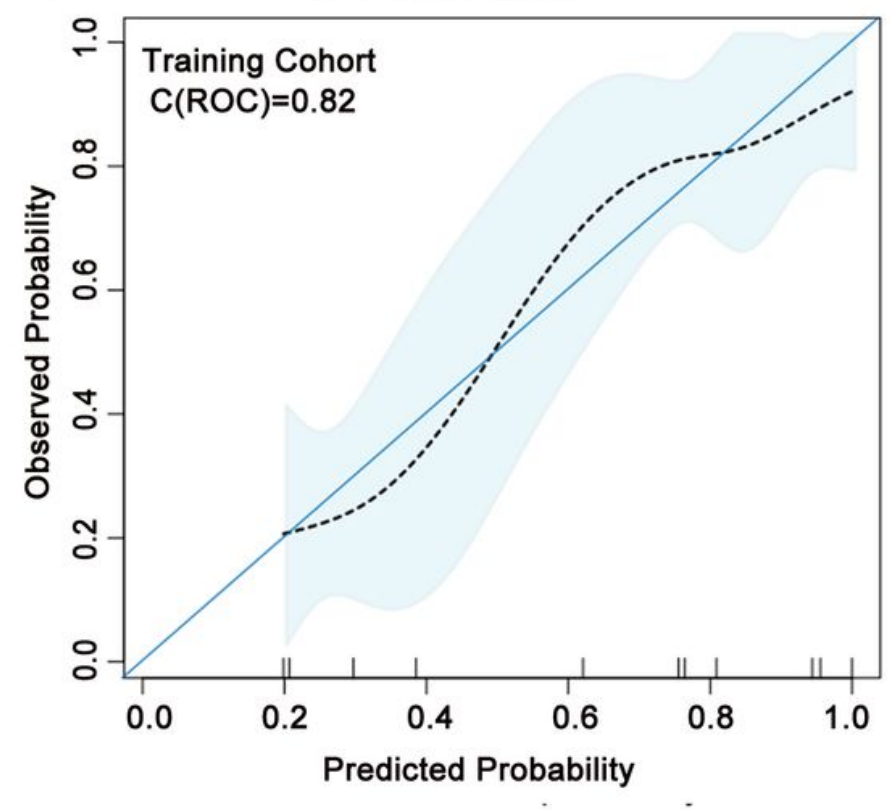

b

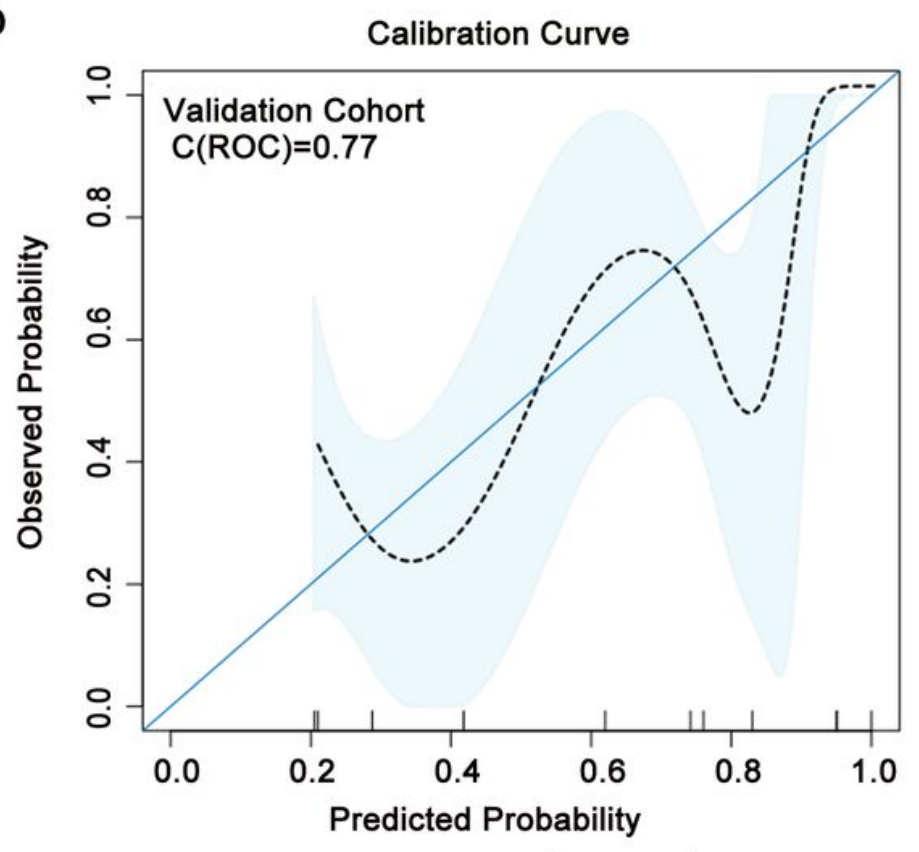

Figure 5

The calibration plot of nomogram. a, The calibration plot shows the prediction performance of the proposed nomogram in the training cohort $(n=126)$. $b$, Validity of the predictive performance of the nomogram in estimating the risk of OS in the validation cohort $(n=89)$. The curve closer to the $45^{\circ}$ line (blue line) indicates the better performance for both $a$ and $b$.

\section{Supplementary Files}

This is a list of supplementary files associated with this preprint. Click to download.

- SupplementaryFigure2.jpg

- SupplementaryTable3.doc

- SupplementaryFigure1copy.jpg

- SupplementaryTable2.doc

- SupplementaryTable1.doc 